\title{
Research Paper \\ Comparison of Postural Control Between Different Age Groups of Girls With Intellectual Disability
}

\author{
Zahra Habibi Masouleh $^{1^{*}}$, , Ali Shamsi Majalani ${ }^{1}$, Parisa Sedaghati ${ }^{1}$
}

1. Department of Sport Sciences, Faculty of Physical Education, University of Guilan, Rasht, Iran.

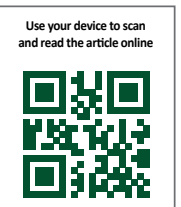

Citation: Habibi Masouleh Z, Shamsi Majalani A, Sedaghati P. [Comparing the Postural Control Among Different Age Ranges in Intellectually Disabled Girls (Persian)]. Journal of Sport Biomechanics. 2021; 6(4):240-249. https://doi.org/10.32598/biomechanics.6.3.3

https://doi.org/10.32598/biomechanics.6.3.3

Key words:

Sensory systems manipulation, Postural control, Intellectual disability, Age groups

\section{ABSTRACT}

Objective The control ability different positions of body in space was due to interaction between neural, sensory, and muscle-skeletal systems, which is generally defined as postural control. Limitations in mobility: The prevalence of balance and gait problems is also high in persons with intellectual disabilities. Thus, the present research aimed to examine the effect of visual, proprioception, and vestibular systems manipulation on postural control in three groups of girls with Intellectual Disability.

Methods The method of the present study is comparative-causative. The statistical population of this study was all girls with oral disabilities in special schools in Rasht. Sixty girls with Intellectual Disability were assessed in groups of 7-9, 10-12, and 18-16 years old ( $N=20)$. Postural control was evaluated in four conditions: open and closed eyes and on hard and soft surfaces. Collected data were analyzed using one-way ANOVA and post hoc test via SPSS software, V. 21 ( $P<0.05)$.

Results The research findings indicated that there were significant differences in postural control in all of the three groups. Based on the results in conditions 1, 2, and 3, there are significant differences between groups 1 and 3 and 2 and 3 .

Conclusion These results support recent findings suggesting that Sensory systems for children with Intellectual Disabilities up to the age of 16-18 are still growing and maturing in terms of organizing and integrating with other systems in postural control. In each of the three age groups, the central nervous system, based on the proprioception information, provided better postural control.

\section{Extended Abstract}

\section{Introduction}

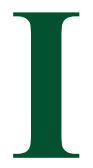

ntellectual Disability (ID) is a complex disorder that is difficult to define precisely. This disorder significantly impairs the functioning of affected people in all areas of their lives and affects their minds and spirit. This disorder disrupts both the "self-concept" and "interpersonal relationships" approaches and significantly reduces the quality of coexistence of these individuals in society [1]. Research- ers study the human development process from different aspects, which has led to creating different areas for further research. One of the most important fields of study created in this area includes body postural control [3].

The ability to control different body positions in space is due to the complex interaction of the nervous, sensory, and musculoskeletal systems, which is generally defined as the postural control system. Movement limitations and problems with balance and gait are high in people with intellectual disabilities [2]. On the other hand, for most functional tasks, the body's vertical orientation

\section{* Corresponding Author:}

Zahra Habibi Masouleh, MSc.

Address: Department of Sport Sciences, Faculty of Physical Education, University of Guilan, Rasht, Iran

Tel: +98 (939) 8036564

E-mail: habibi.z1992@gmail.com 
Table 1. Comparison of the mean postural control of the subjects in 4 assessed situations among age groups

\begin{tabular}{|c|c|c|c|c|}
\hline Variables & Groups & Mean士SD & $\mathbf{F}$ & $\mathbf{P}^{*}$ \\
\hline \multirow{3}{*}{$\begin{array}{l}\text { First sensory position } \\
\qquad \text { C1 }\end{array}$} & $7-9$ & $3.75 \pm 1.20$ & \multirow{3}{*}{3.321} & \multirow{3}{*}{$0.043^{*}$} \\
\hline & $10-12$ & $3.10 \pm 1.20$ & & \\
\hline & $16-18$ & $2.85 \pm 0.99$ & & \\
\hline \multirow{3}{*}{$\begin{array}{l}\text { Second sensory position } \\
\text { C2 }\end{array}$} & $7-9$ & $6.10 \pm 1.11$ & \multirow{3}{*}{5.884} & \multirow{3}{*}{$0.005^{*}$} \\
\hline & $10-12$ & $6.05 \pm 1.32$ & & \\
\hline & $16-18$ & $5.00 \pm 0.97$ & & \\
\hline \multirow{3}{*}{$\begin{array}{l}\text { Third sensory position } \\
\text { C3 }\end{array}$} & $7-9$ & $8.00 \pm 1.45$ & \multirow{3}{*}{6.625} & \multirow{3}{*}{$0.003^{*}$} \\
\hline & $10-12$ & $7.55 \pm 1.43$ & & \\
\hline & $16-18$ & $6.50 \pm 1.10$ & & \\
\hline \multirow{3}{*}{$\begin{array}{l}\text { Fourth sensory position } \\
\text { C4 }\end{array}$} & $7-9$ & $8.25 \pm 1.33$ & \multirow{3}{*}{13.936} & \multirow{3}{*}{$0.001^{*}$} \\
\hline & $10-12$ & $7.10 \pm 1.37$ & & \\
\hline & $16-18$ & $6.00 \pm 1.34$ & & \\
\hline
\end{tabular}

must be maintained; therefore, several sensory systems are used in this process. The vestibular system is used to control the force of gravity. The proprioceptive system is used to control the base of support, and the visual system is used to control the relationship between the body and objects in the environment [5]. Therefore, this study aimed to compare postural control between different age groups in girls with intellectual disabilities.

\section{Methods}

The present study was a causal-comparative study whose data were quantitatively measured. Measurements were performed in exceptional schools of students with mental disabilities in Gilan Province. It should be noted that all measurements were performed equally. The statistical population of this study included all female students with intellectual disabilities in Gilan Province. Due to the limitations in selecting subjects, this study's statistical sample included 60 female students with intellectual disabilities in the age groups of 7-9, 10-12, and 16-18 years who were randomly and purposively selected and evaluated. Then, to evaluate the subjects' ability to control their standing position, a postural control test was performed on them in four different sensory positions.

The four sensory positions in this test were as follows: the first position, standing on one foot on a stable surface with open eyes; the second position, standing on one foot on an unstable surface with open eyes and head hyperextension; the third position, standing on one foot on a stable surface with eyes closed and head hyperextension; the fourth position, standing on one foot on an unstable surface with eyes closed. Each stage of the test was performed in 20 seconds, and the number of errors made was recorded as the subject's score in that stage. In this study, the Shapiro-Wilk test was used to evaluate the data's normality, and descriptive statistics indices (Mean \pm SD) were used to describe the data. Inferential statistical indices (such as one-way ANOVA and Bonferroni post hoc test) were used to analyze the data by SPSS software, V. 21 at the level of $\mathrm{P}<0.05$.

\section{Results}

To analyze postural control data in the study groups, the Bonferroni post hoc test was used. This test confirmed no sensory interference in the first position, and all three sensory systems of the subject (visual, vestibular, and proprioceptive systems) were available.

The results of the three groups' significant differences in postural control scores were $\mathrm{P}=0.043, \mathrm{P}=0.005, \mathrm{P}=0.003$, $\mathrm{P}=0.000$. Based on the results, there was a significant difference between the first, second, and third positions between the first and third groups and between the second and third groups $(\mathrm{P}<0.05)$. 


\section{Discussion and Conclusion}

According to the results of this study, in children with intellectual disabilities, sensory systems in terms of organization and integration continue to develop and mature until the age of 16-18, and the central nervous system, by receiving information from the proprioceptive system, provides better postural control. This study revealed a significant difference between the first and third groups in the first position, where no sensory manipulation was performed, and all three systems were available to the individual. Observation of postural control scores in Table 1 confirmed that the development and maturity of the proprioceptive and visual systems were insignificant in the first and second groups, but in the third group, there was significant development and maturity.

However, the vestibular system's rate of development and maturity was noticeable among all three age groups. Since postural control scores were not the same in all four positions in the three age groups, the results obtained in this section were consistent with different results. The results of this study and other similar studies confirm that each of the visual, somatosensory, and vestibular systems that play an essential role in postural control in people with intellectual disabilities are constantly developing and maturing (up to the age of 16-18) in terms of organization and integration with other postural control systems. On the other hand, in all three age groups studied, the central nervous system provided better postural control based on somatosensory information.

\section{Ethical Considerations}

Compliance with ethical guidelines

All ethical principles are considered in this article. The participants were informed about the purpose of the research and its implementation stages. They were also assured about the confidentiality of their information and were free to leave the study whenever they wished, and if desired, the research results would be available to them.

\section{Funding}

The paper was extracted from the MA. thesis of the first author, Department of Sport Sciences, Faculty of Physical Education, University of Guilan, Rasht.Also, This study was conducted in collaboration with the Exceptional Education Department of Gilan Province.

Authors' contributions

All authors equally contributed to preparing this article.

\section{Conflicts of interest}

The authors declared no conflict of interest.

\section{Acknowledgement}

All author would like to thank all the participants and the principles of exceptional schools in Gilan province for their collaboration. 


\title{
مقايسه كنترل ياسجر بين ردههاى سنى مختلف در دختران كمتوان ذهنى
}

\author{
"زهرا حبيبى ماسوله' (ن)، على شمس ماجلانى'، بريسا صداقتى' \\ 1. كروه آسيبشناسى ورزشى و حركات اصلاحى، دانشكده تربيت بدنى و علوم ورزشى، دانشكاه كيلان، رشت، ايران.
}

\begin{abstract}
حكيد

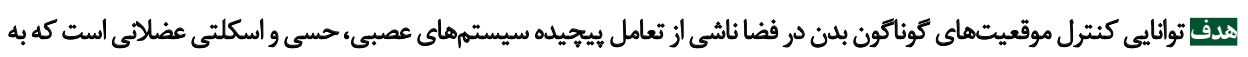

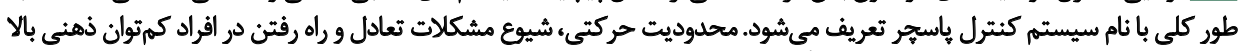

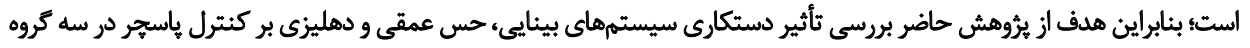
از دختران كمتوان ذهنى بود.

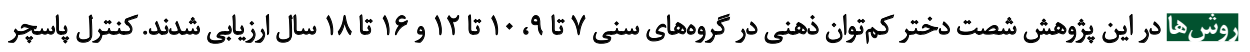

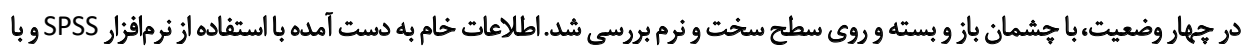

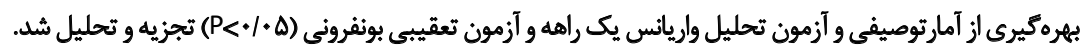

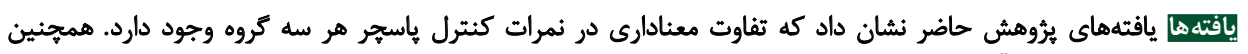

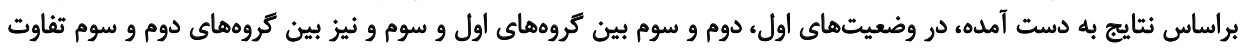

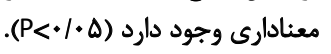

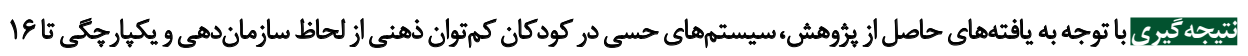

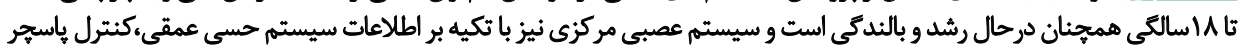

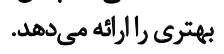

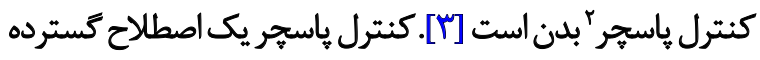

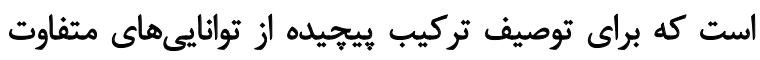

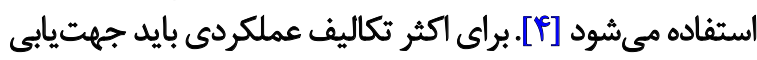

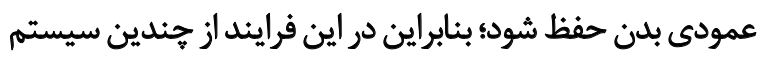

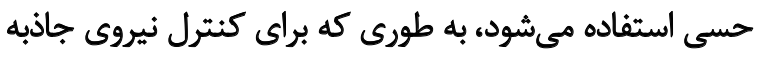

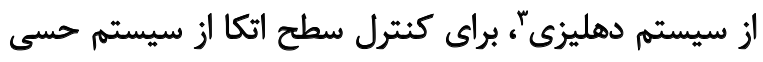

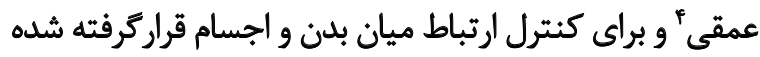

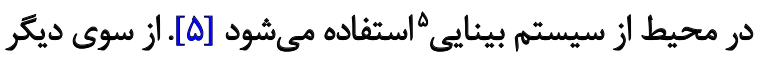

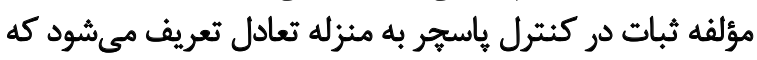

توانايى كنترل توده بدن در ارتباط با سطح اتكا است [ع، هـ]. تغييرات رشدى در كنترل پاسجر در طول سالهاى اول
2. Postural Control
3. Vestibular
4. Proprioceptive
5. Visual

1. Intellectual disability

مقدمه

كمتوانى ذهنى 'يك اختلال بيجيجيده است كه تعريف آن به آنه

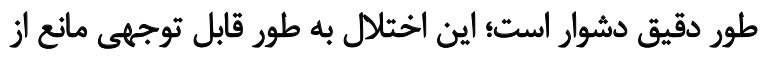

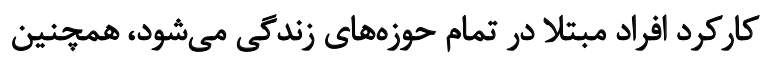

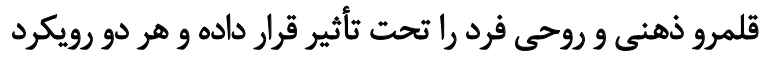

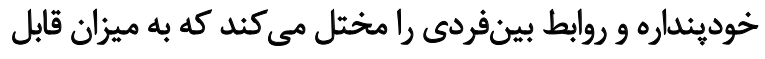

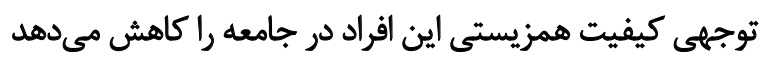

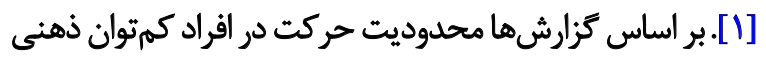

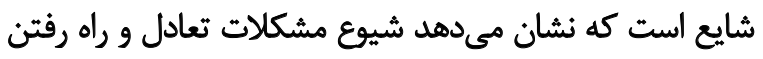

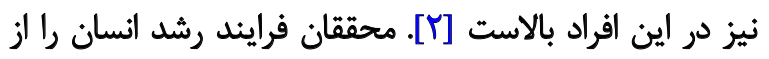

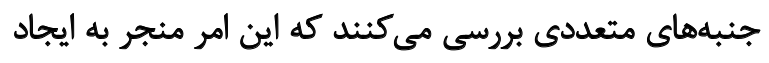

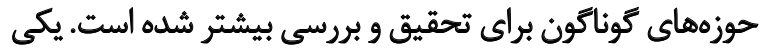

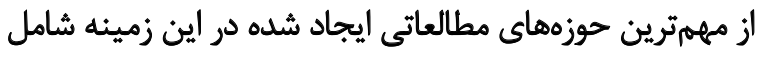

$$
\text { ……. }
$$


جندكائه عصبى، حسى و حسى حركتى (مانند عصب مركزي،

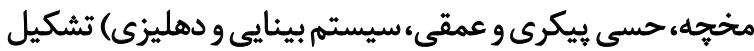

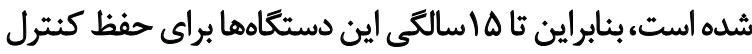

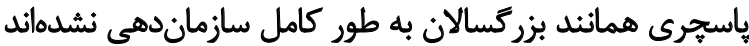

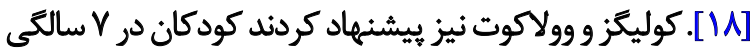

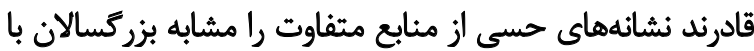

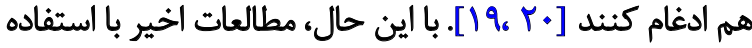

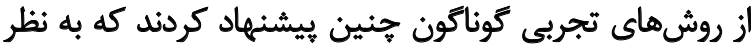

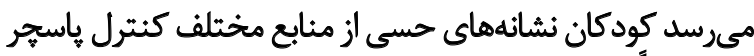

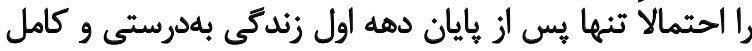

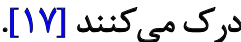

مطالعات اخير نشان دادهاند كه توسعه كنترل ياسجر ممكن

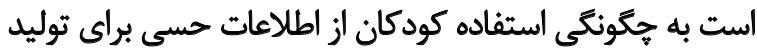

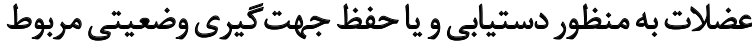

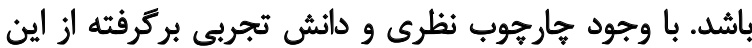

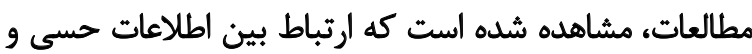

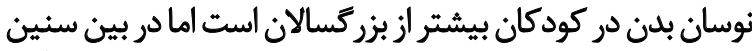

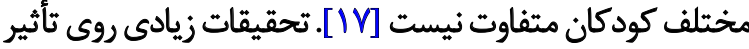

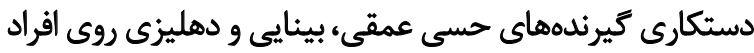

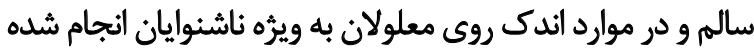

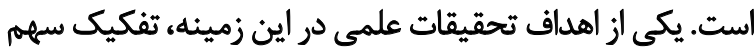

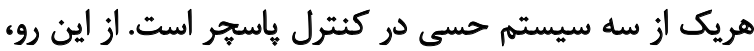

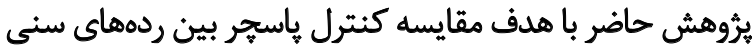
مختلف در دختران كمتوان ذهنى انجام كرفت

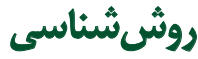

هرؤشش حاضر از نوع علتى مقايسهاى بوده كه دادههاى آن به آنه

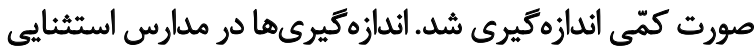

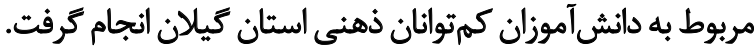

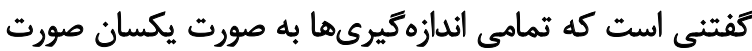

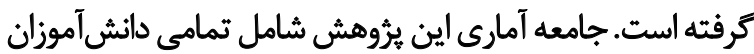

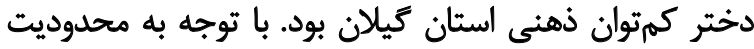

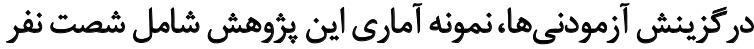

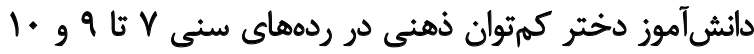

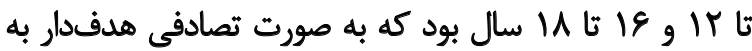

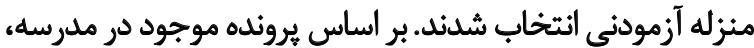

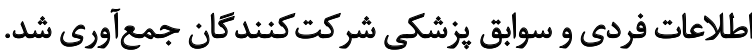

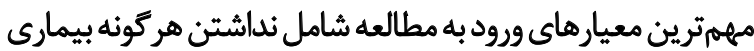

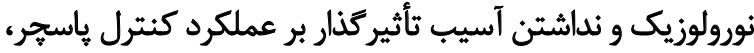

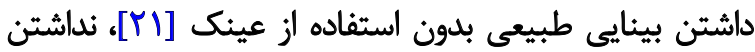

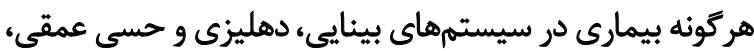

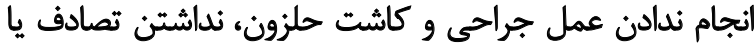

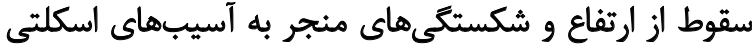

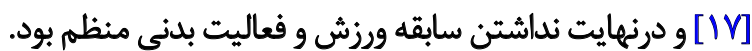

زندكى رخ مى دهد اما مكانيسمهاى زيربنايى اين تغييرات هنوز

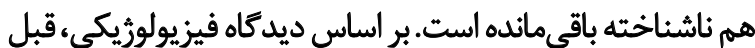

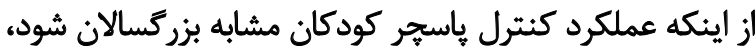

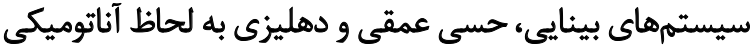

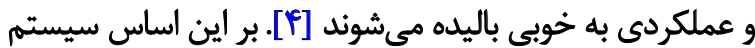

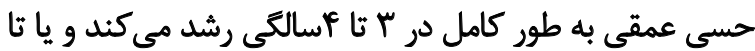

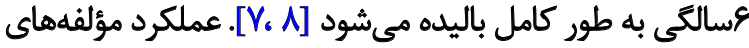
سيستم دهليزى (شامل مجارى نيمدايره، اندامهاى اتوليتئي و

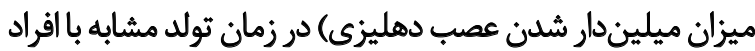

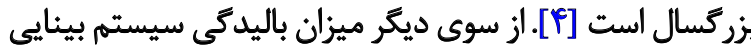

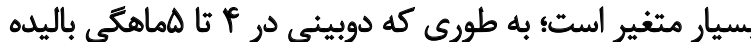

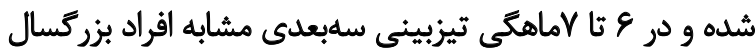

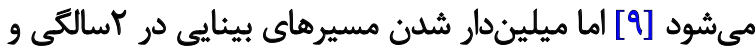

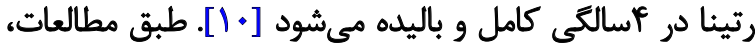

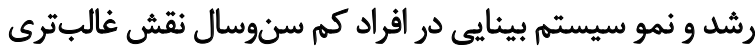

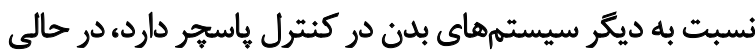

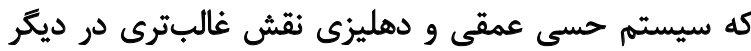

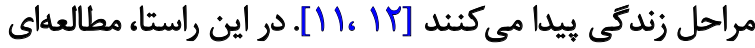

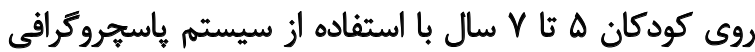

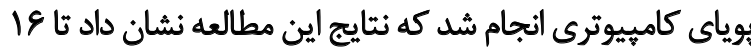

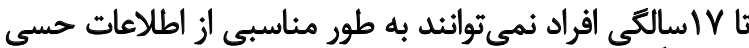

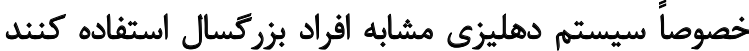

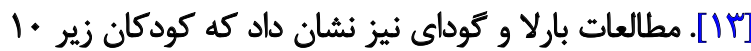

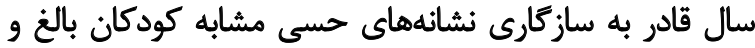

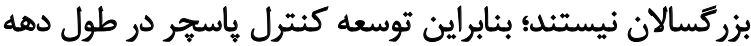

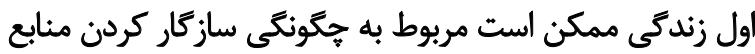

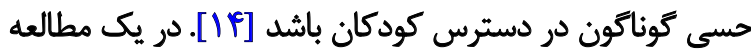

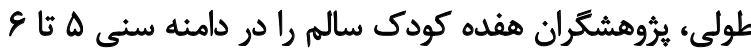

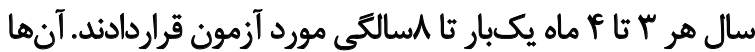

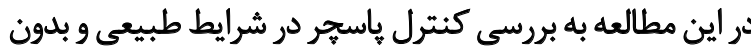

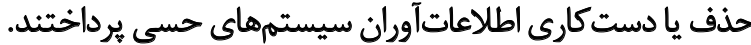

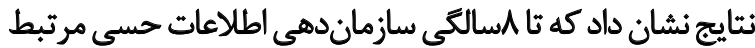

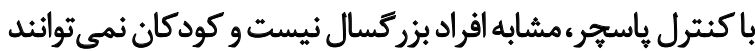

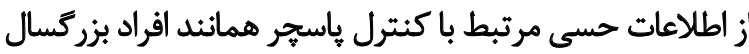

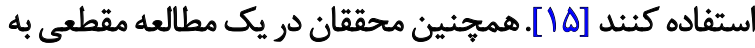

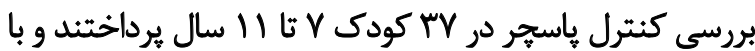

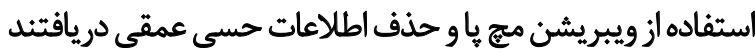

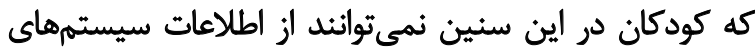

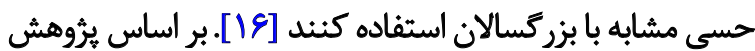

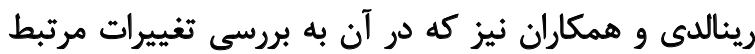

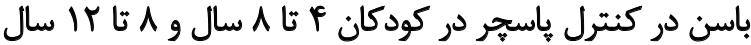

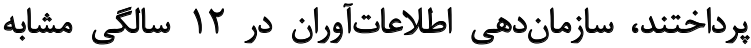

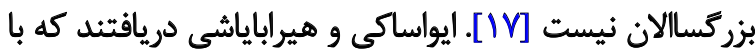

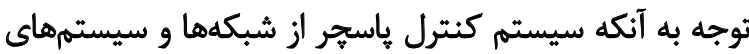


مى شود. در حالت سوم نيز دادههاى بينايى و دهليزى مختل شده

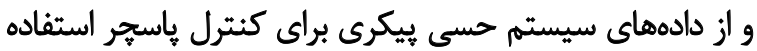

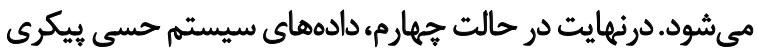

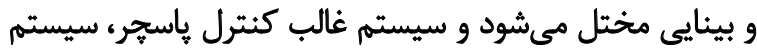

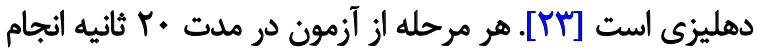

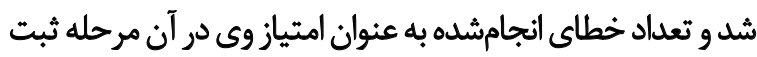

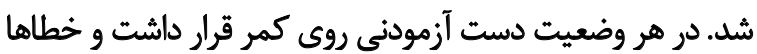

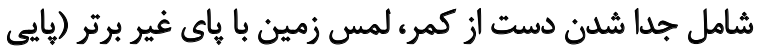

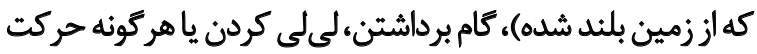

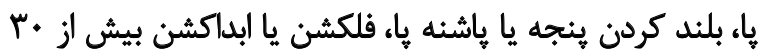

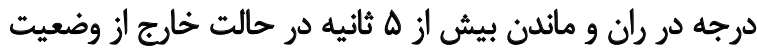

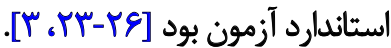

در مطالعه حاضر از آزمون شايِيرو ويلك براى ارزيابى نرمال

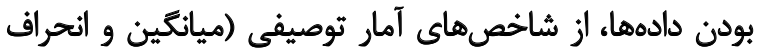

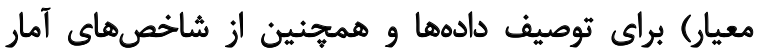

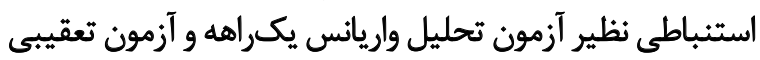

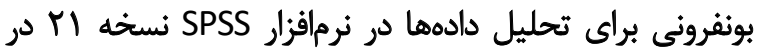

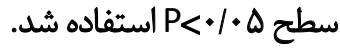

ثتايج

با توجه به جدول شماره ا نتايج كنترل ياسجر آزمودنىها

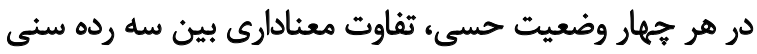

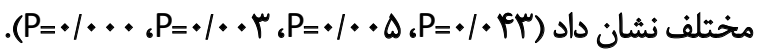
به منظور تجزيه و تحليل دادهاى كنترل ياسجر در كروههاى
ابتدا از والدين شركت كنيدكان رضايتنامه كتبى براى شركت

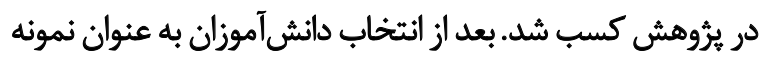

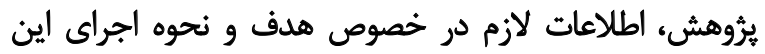

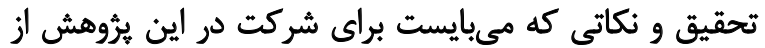
طرف شركت كنند

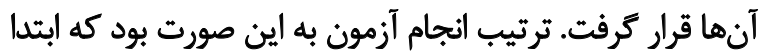

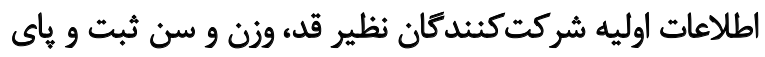

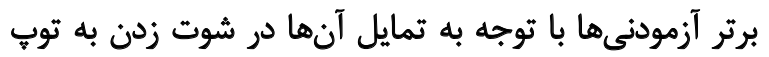

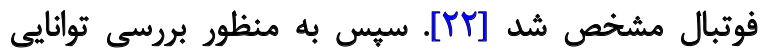

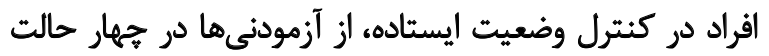

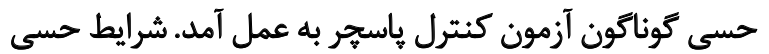
جهاركانه در اين آزمون بدين شرح استرل ياسجر به عمل

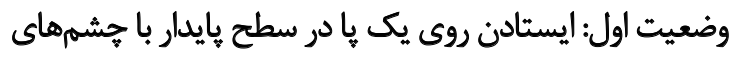
باز؛

وضعيت دوم: ايستادن روى يك يا در سطح نإيايدار با حشمهاى

$$
\text { باز و هاييراكستنشن سر: إنست }
$$

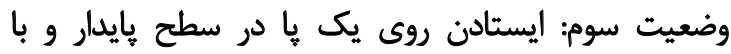
جشمهاى بسته و هايبراكستنشن سر: ئي

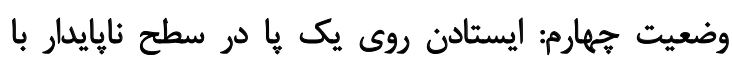
جشهمهاي بسته. در حالت اول، هرسه سيستم حسى دركير در كنترل بِاسجر

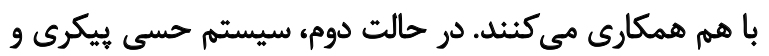

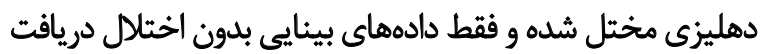

جدول ا. مقايسه ميانكين كنترل ياسجير آزمودنىهاى بُروهش در جهار وضعيت ارزيابىشده بين كروهاي سنى

\begin{tabular}{|c|c|c|c|c|}
\hline P* & $\mathbf{F}$ & هيانغين ثانحراف استاندارد & $\log$ & متغير \\
\hline \multirow{3}{*}{ H } & \multirow{3}{*}{$r / M T I$} & $T / V \Delta \pm 1 / T$. & $9-V$ & \multirow{3}{*}{ وضعيت حسى اول } \\
\hline & & $r / 1+ \pm 1 / r$. & $\mid r-1$ & \\
\hline & & $T / A \Delta \pm \cdot / 99$ & $1 A-18$ & \\
\hline \multirow{3}{*}{$\% \infty$} & \multirow{3}{*}{$\Delta / M P$} & $8 / 1 \cdot \pm 1 / 11$ & $9-\vee$ & \multirow{3}{*}{ وضعيت حسى دوم } \\
\hline & & $8 / \cdot \Delta \pm / / T r$ & $1 r-1$. & \\
\hline & & ه/..土*/q४ & $14-18$ & \\
\hline \multirow{3}{*}{$1 \cdot+r$} & \multirow{3}{*}{ s/ETo } & $N \cdot \pm \pm / / \uparrow \omega$ & $9-Y$ & \multirow{3}{*}{ وضعيت حسى سوم } \\
\hline & & $V / \Delta \Delta \pm 1 / \Gamma T$ & $\mid r-1$. & \\
\hline & & $8 / 0+ \pm 1 / 1$ & $11-18$ & \\
\hline \multirow{3}{*}{$.1 .+1$} & \multirow{3}{*}{ Ir/arg } & $N T \Delta \pm 1 / M r$ & $9-Y$ & \multirow{3}{*}{ وضعيت حسى جهارم } \\
\hline & & $V / 1 \cdot \pm 1 / T V$ & $\mid r=1$ & \\
\hline & & $9 / . \pm 1 / m e$ & $1 A-18$ & \\
\hline
\end{tabular}


جدول r. اختلاف ميانكين در كروههاى مختلف در وضعيت اول ارزيابى

\begin{tabular}{|c|c|c|c|c|}
\hline $\mathbf{P}$ & تفاوت ميانكين & كروه & كروه & متغير \\
\hline.$/ \pi$ & .190 & $\mid r-1$. & \multirow{2}{*}{$q-\gamma$} & \multirow{3}{*}{ حالت اول } \\
\hline $.1 .+9 \varphi^{\circ}$ &.$/ 9$. & $\mid A-18$ & & \\
\hline$v /$. &.$/ T \Delta$ & $\mid A-18$ & $\mid r-1$. & \\
\hline $1 / \cdots$ & .1 .0 & $\mid r-1$. & \multirow{2}{*}{$9-\gamma$} & \multirow{3}{*}{ حالت دوم } \\
\hline$\cdot 1 / \cdot 111^{*}$ & $1 / .1$ & $\mid A-18$ & & \\
\hline $.1 .16^{\circ}$ & $1 / \cdot \Delta$ & $|A-| S$ & $\mid r-1$. & \\
\hline . IAva &.$/ F \Delta$ & $\mid r-1$. & \multirow{2}{*}{$9-Y$} & \multirow{3}{*}{ حالت سوم } \\
\hline$\%$ & $V / \Delta$. & $|A-| s$ & & \\
\hline $1 .+41^{\circ}$ & $1 / \cdot 0$ & $\mid A-18$ & $\mid r-1$. & \\
\hline$\%$ & $1 / 10$ & $1 r-1$. & \multirow{2}{*}{$9-Y$} & \multirow{3}{*}{ حالت جهارم } \\
\hline $.4 / .+)^{*}$ & T/TA & $\mid A-18$ & & \\
\hline . & $1 / 1$. & $1 \mathrm{~A}-18$ & $\mid r-1$. & \\
\hline
\end{tabular}

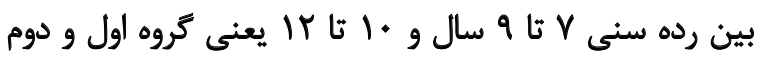

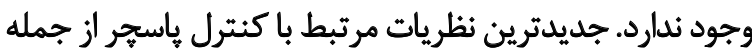

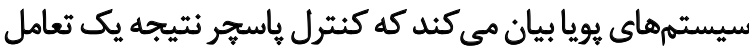

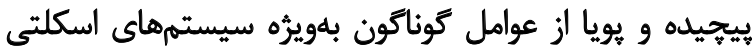

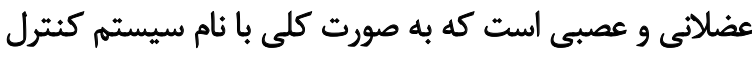

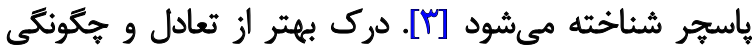

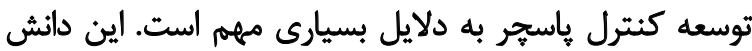

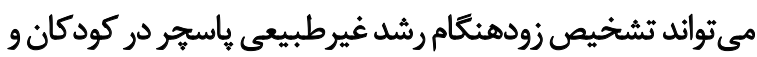

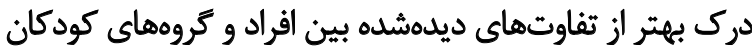

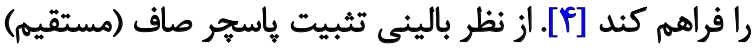

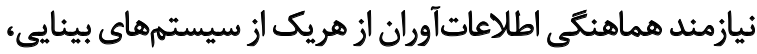

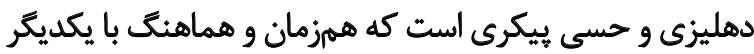

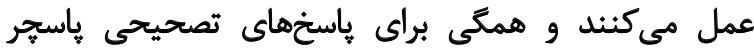

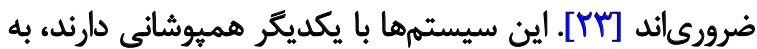

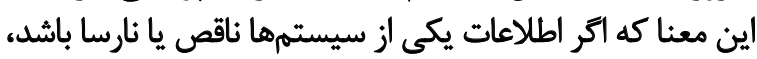

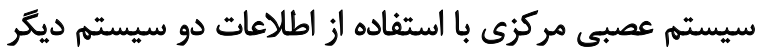

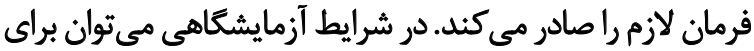

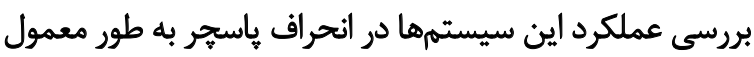

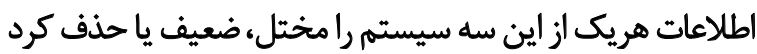

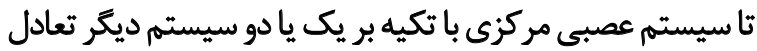

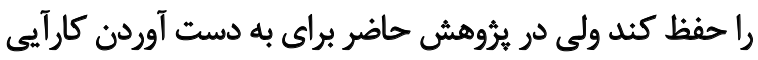

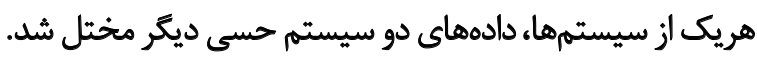

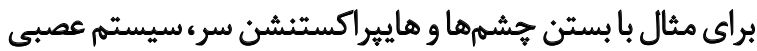

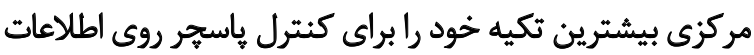

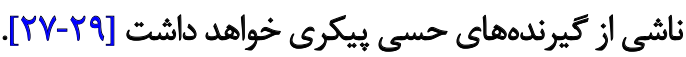

نتايج يثوهش حاضر نشان داد در وضعيت اول كه هيجكونه
مورد مطالعه، از آزمون تعقيبى بونفرونى استفاده شد. نتايج اين

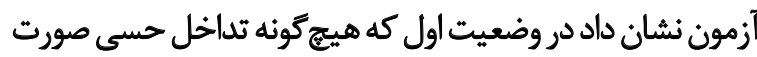

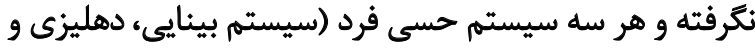

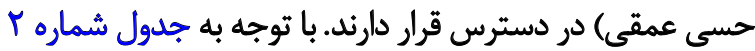

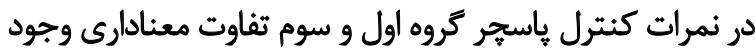

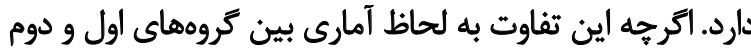

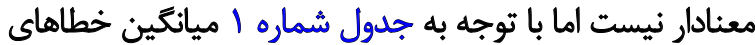

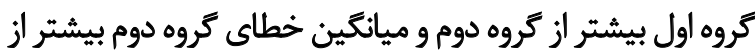

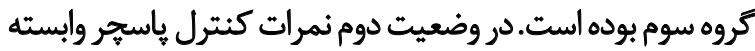

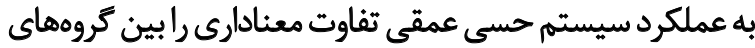

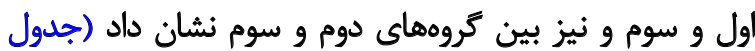

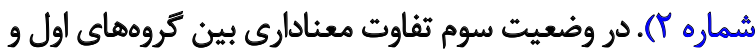

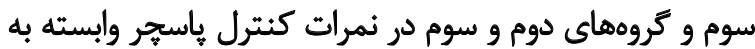

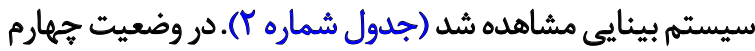

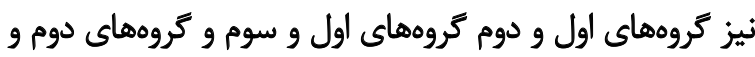

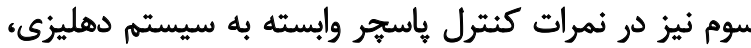

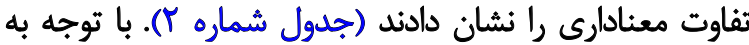

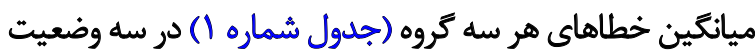

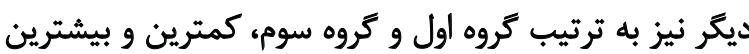
نمره را در كنترل ياسجر كسب كردني كروند

بحث

نتايج اين يُروهش نشان داد كه كنترل ياسجِر آزمودنى هادر هر

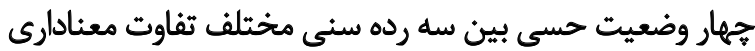

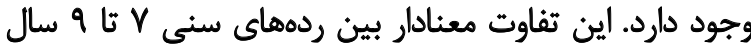

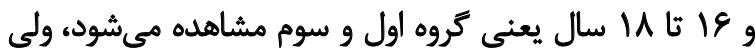


ارائه دادند. يُيشنهاد مى شود در ساعات ورزش اين افراد در مدارس

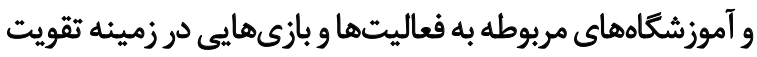

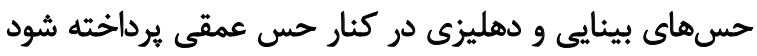

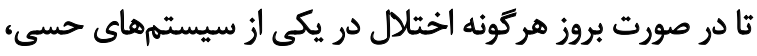

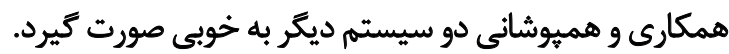

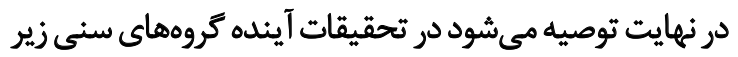

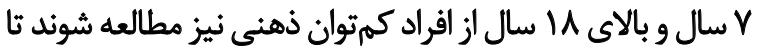

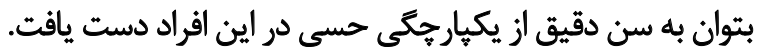

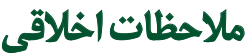 \\ بيروى از اصول اخلاق بؤوهش}

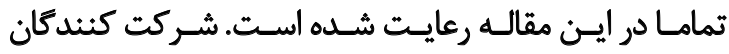

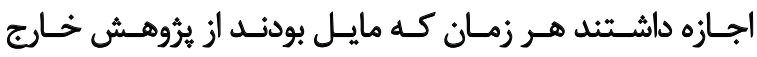

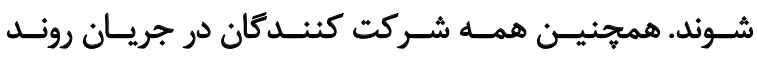

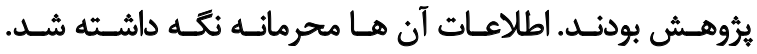

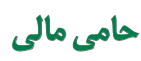

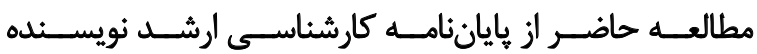

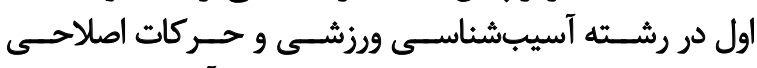

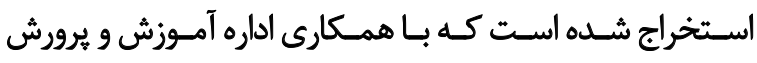

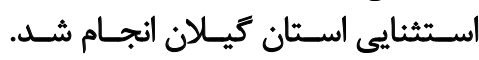

$$
\text { مشاركت نويسند مَّان }
$$

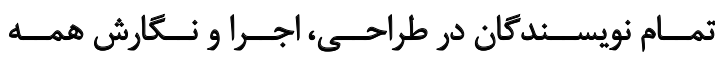

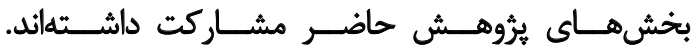

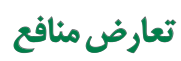

بنابر اظهار نويسندكان، اين مقاله تعارض منافع ندارد.

$$
\text { تشكر و قدردانى }
$$

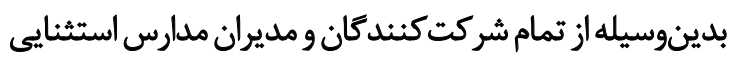

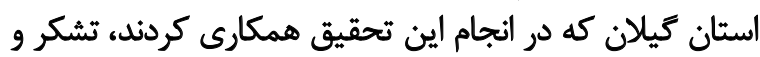

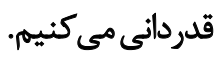

دستكارى حسى صورت نكَرفته و هر سه سيستم در دسترس

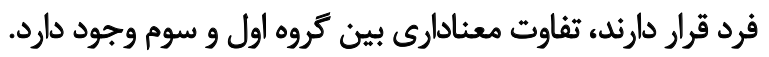

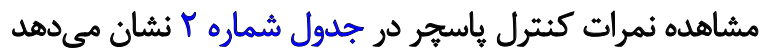

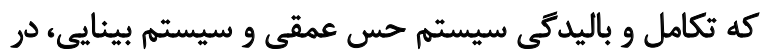

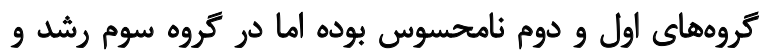

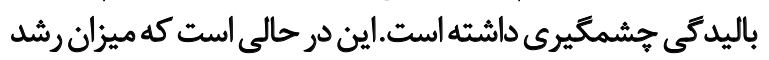

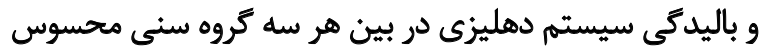

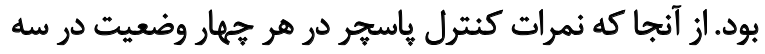

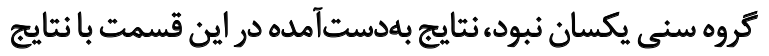

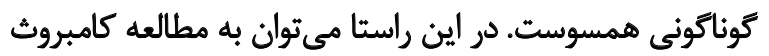

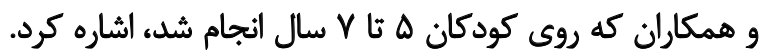

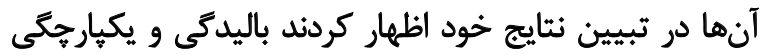

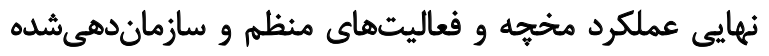

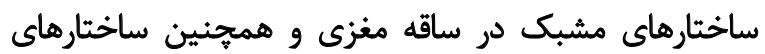

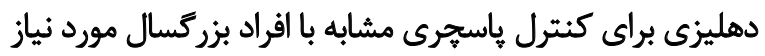

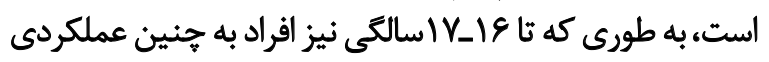

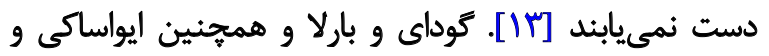

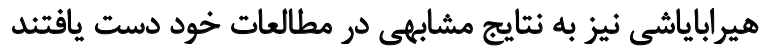

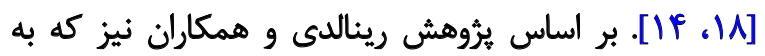

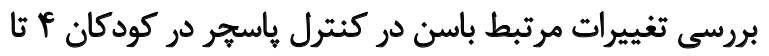

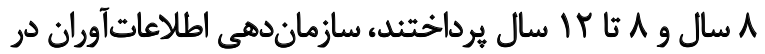

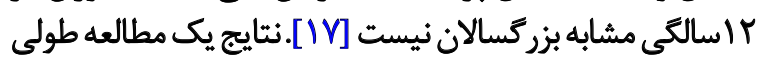

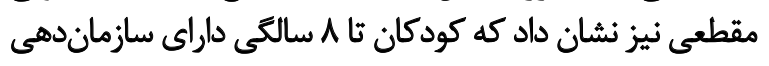

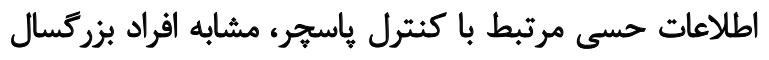

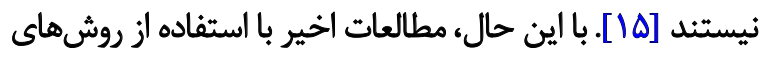

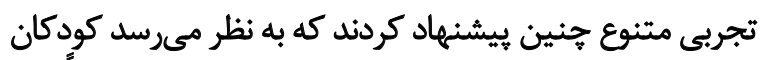

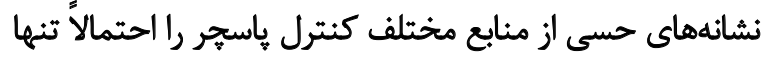

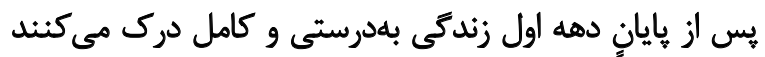

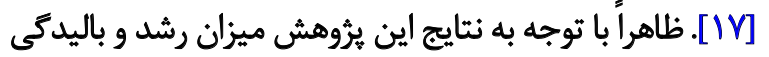

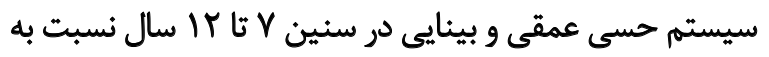

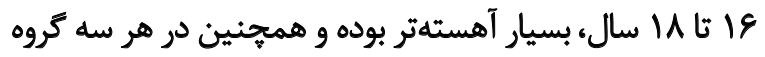

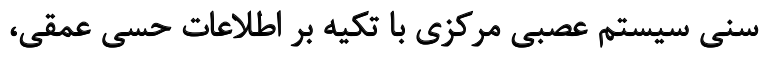

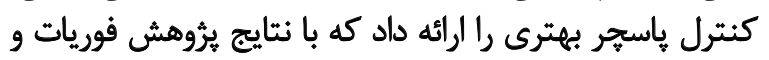

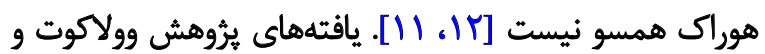

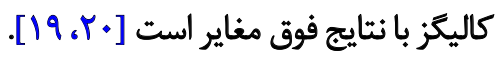

\section{نتيجه كيرى نهايى}

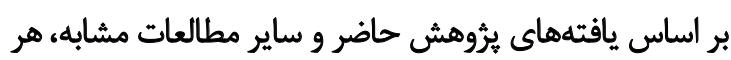

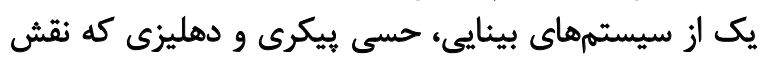

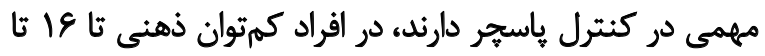

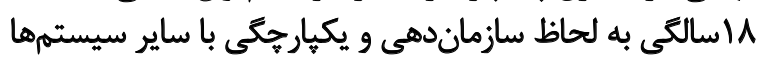

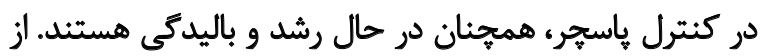

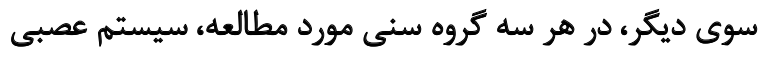

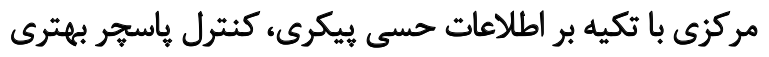




\section{References}

[1] Jankowicz-Szymanska A, Mikolajczyk E, Wojtanowski W. The effect of physical training on static balance in young people with intellectual disability. Res Dev Disabil. 2012; 33(2):675-81. [DOI:10.1016/j. ridd.2011.11.015] [PMID]

[2] World Health Organization. International classification of functioning, disability and health: ICF. Geneva: World Health Organization; 2001. https://www.who.int/standards/classifications/international-classification-of-functioning-disability-and-health

[3] Shams A, Aslankhani MA, Abdoli B, Ashayeri H, Namazi Zadeh M. The effect of visual, proprioception and vestibular systems manipulation on postural control in boys with 4-16 years-old. J Shahrekord Uni Med Sci. 2014; 16(3):22-32. http://journal.skums.ac.ir/browse.php?a_ id $=1802 \&$ sid= $1 \&$ slc_lang=en

[4] Peterson ML, Christou E, Rosengren KS. Children achieve adult-like sensory integration during stance at 12-years-old. Gait Posture. 2006; 23(4):455-63. [DOI:10.1016/j.gaitpost.2005.05.003] [PMID]

[5] Shumway-Cook A, Woollacott MH. Motor control: Translating research into clinical practice. Philadelphia: Lippincott Williams \& Wilkins; 2007.

[6] Winter DA, Patla AE, Prince F, Ishac M, Gielo-Perczak K. Stiffness control of balance in quiet standing. J neurophysiol. 1998; 80(3):1211-21. [DOI:10.1152/jn.1998.80.3.1211] [PMID]

[7] Steindl R, Kunz K, Schrott-Fischer A, Scholtz AW. Effect of age and sex on maturation of sensory systems and balance control. Dev Med Child Neurol. 2006; 48(6):477-82. [DOI:10.1111/j.1469-8749.2006.tb01299.x] [PMID]

[8] Fitzpatrick R, McCloskey DI. Proprioceptive, visual and vestibular thresholds for the perception of sway during standing in humans. J physiol. 1994; 478(Pt 1):173-86. [DOI:10.1113/jphysiol.1994.sp020240] [PMID] [PMCID]

[9] Neuringer M, Jeffrey BG. Visual development: Neural basis and new assessment methods. J Pediatr. 2003; 143(4 suppl):S87-95. [DOI:10.1067/ S0022-3476(03)00406-2] [PMID]

[10] Brecelj J. From immature to mature pattern ERG and VEP. Doc Ophthalmol. 2003; 107(3):215-24. [DOI:10.1023/
[D. B:DOOP.0000005330.62543.9c] [PMID]

[11] Horak FB, Henry SM, Shumway-Cook A. Postural perturbations: new insights for treatment of balance disorders. Phys Ther. 1997; 77(5):51733. [DOI:10.1093/ptj/77.5.517] [PMID]

[12] Foudriat BA, Di Fabio RP, Anderson JH. Sensory organization of balance responses in children 3-6 years of age: A normative study with diagnostic implications. Int J Pediatr Otorhinolaryngol. 1993; 27(3):255-71. [DOI:10.1016/0165-5876(93)90231-Q] [PMID]

[13] Cumberworth VL, Patel NN, Rogers W, Kenyon GS. The maturation of balance in children. J Laryngol Otol. 2007; 121(5):449-54. [DOI:10.1017/ S0022215106004051] [PMID]

[14] Godoi D, Barela JA. Body sway and sensory motor coupling adaptation in children: Effects of distance manipulation. Dev Psychobiol. 2008; 50(1):77-87. [DOI:10.1002/dev.20272] [PMID]

[15] Kirshenbaum N, Riach C, Starkes J. Non-linear development of postural control and strategy use in young children: A longitudinal study. Exp Brain Res. 2001; 140(4):420-31. [DOI:10.1007/s002210100835] [PMID]

[16] Cuisinier R, Olivier I, Vaugoyeau M, Nougier V, Assaiante C. Reweighting of sensory inputs to control quiet standing in children from 7 to
11 and in adults. PLoS One. 2011; 6(5):e19697. [DOI:10.1371/journal. pone.0019697] [PMID] [PMCID]

[17] Rinaldi NM, Polastri PF, Barela JA. Age-related changes in postura control sensory reweighting. Neurosci Lett. 2009; 467(3):225-9. [DOI:10.1016/j.neulet.2009.10.042] [PMID]

[18] Hirabayashi SI, Iwasaki Y. Developmental perspective of sensory organization on postural control. Brain Dev. 1995; 17(2):111-3. [DOI:10.1016/0387-7604(95)00009-Z] [PMID]

[19] Woollacott M, Debû B, Mowatt M. Neuromuscular control of posture in the infant and child: is vision dominant? J Mot Behav. 1987; 19(2):16786. [DOI:10.1080/00222895.1987.10735406] [PMID]

[20] Shumway-Cook A, Woollacott MH. The growth of stability: Postural control from a developmental perspective. J Mot Behav. 1985; 7(2):13147. [DOI:10.1080/00222895.1985.10735341] [PMID]

[21] Ferber-Viart C, lonescu E, Morlet T, Froehlich P, Dubreuil C. Balance in healthy individuals assessed with Equitest: Maturation and normative data for children and young adults. Int J Pediatr Otorhinolaryngol. 2007; 71(7):1041-6. [DOI:10.1016/j.ijporl.2007.03.012] [PMID]

[22] Farzaneh Hessari A, Daneshmandi H, Mahdavi S. [The effect of 8 weeks of core stabilization training program on balance in hearing impaired students (Persian)]. J Sport Med. 2011;3(2):67-83. https://jsmed. ut.ac.ir/article_24966.html\#: :text=The\%20results\%20indicated\%20 a\%20significant,0.05).\&text=In\%20conclusion\%2C\%20core\%20stabilization\%20training,along\%20with\%20other\%20training\%20programs.

[23] Seyedi M, Seidi F, Minoonejad H. [An Investigation of the efficiency of sensory systems involved in postural control in deaf athletes and nonathletes (Persian)]. J Sport Med. 2015; 7(1):111-27. https://www.sid.ir/ en/journal/ViewPaper.aspx?id=463348

[24] Parvizi S. [Comparison of balance between 6-12 year old deaf boys and their relationship with age (Persian)] [MSc. Thesis]. Tehran: Iran University of Medical Sciences; 2001.

[25] Sedaghati P, Zolghare H, Shahbazi M. The effect of proprioceptive, vestibular and visual changes on posture control among the athletes with and without medial tibial stress syndrome. Feyz: J Kashan Un Med Sci. 2019; 23(1):68-74. https://feyz.kaums.ac.ir/browse.php?a id $=3631 \&$ sid=1\&slc_lang=en

[26] Taheri M, Irandoust K, Norasteh A, Shaviklo J. [The effect of combined core stability and neuromuscular training on postural control in students with congenital hearing loss (Persian)]. J Res Rehabil Sci. 2017; 13(2):806. http://jrrs.mui.ac.ir/index.php/jrrs/article/view/2846

[27] Ebrahimi Sani S. [Comparison of static equilibrium and the effect of sensory systems on its control in healthy children with developmental coordination disorder (Persian)] [MSc. thesis]. Tehran University of Tehran; 2009. https://lib1.ut.ac.ir:8443/site/catalogue/1385265

[28] Mohammadi F. [Evaluation of CNS function in the control of the hypersecretory and sensory-system manipulation in Gulbal athletes and comparison with non-athletics blind and sighted (Persian)] [MSc. thesis] ]. Tehran: University of Tehran; 2009. https://thesis2.ut.ac.ir/thesis/ UTCatalog/UTThesis/Forms/ThesisBrief.aspx?thesisID=c8b27b1c-11f34fc3-9f0f-61b9416512bc

[29] Szymczyk D, Drużbicki M, Dudek J, Szczepanik M, Snela S. Balance and postural stability in football players with hearing impairment. Young Sport Sci Ukraine. 2012; 3:258-63. https://www.researchgate. net/profile/Magdalena-Szczepanik/publication/285733259_Balance and postural stability in football players with hearing impairment/ links/588280a24585150dde40562f/Balance-and-postural-stability-infootball-players-with-hearing-impairment.pdf 
This Page Intentionally Left Blank 